\title{
Gregor Schöllgen, Krieg. Hundert Jahre Weltgeschichte
}

\section{Philipp Siegert}

\section{OpenEdition \\ Journals}

Édition électronique

URL : http://journals.openedition.org/ifha/11171

DOI : 10.4000/ifha. 11171

ISSN : 2198-8943

Éditeur

IFRA - Institut franco-allemand (sciences historiques et sociales)

\section{Référence électronique}

Philipp Siegert, « Gregor Schöllgen, Krieg. Hundert Jahre Weltgeschichte », Revue de I'IFHA [En ligne],

Date de recension, mis en ligne le 22 mars 2021, consulté le 24 mars 2021. URL : http://

journals.openedition.org/ifha/11171 ; DOI : https://doi.org/10.4000/ifha.11171

Ce document a été généré automatiquement le 24 mars 2021.

(CIFHA 


\title{
Gregor Schöllgen, Krieg. Hundert Jahre Weltgeschichte
}

\author{
Philipp Siegert
}

Gregor Schöllgen, professeur désormais émérite de l'université Erlangen, a présenté à la fin de sa carrière active un ouvrage qui se veut le "portrait d'un monde qui se trouve, depuis 100 ans, au bord de l'abysse" (p. 8). Le titre "Guerre : cent ans d'histoire mondiale »annonce le fil rouge de l'ouvrage, mais pas le thème auquel l'auteur se bornerait dans sa démarche. Si la catégorie de guerre, de conflit dans ses formes variées, reste bien l'angle d'approche tout au long du récit, Gregor Schöllgen offre bien plus qu'une histoire des conflits violents depuis 1917. Il ne s'agit pas d'une simple chronologie explicative des guerres, révolutions, guérillas ou du terrorisme, mais plutôt de la présentation d'un mode d'appréhension possible de notre monde sous deux aspects: l'évolution de la nature des conflits et l'évolution des «grilles de lecture "(géo-)politiques qu'elle a

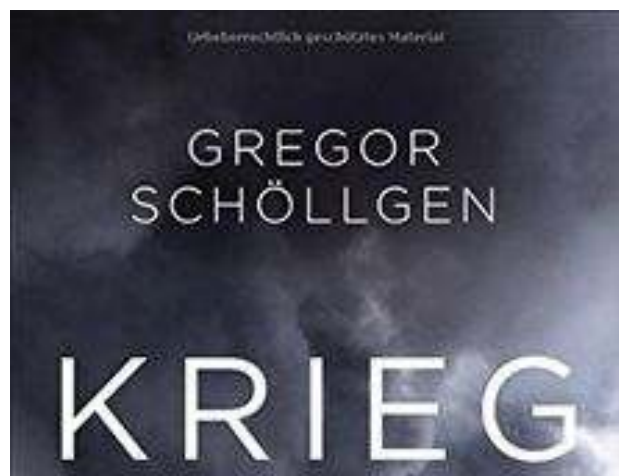

HUNDERT JAHRE WELTGESCHICHTE

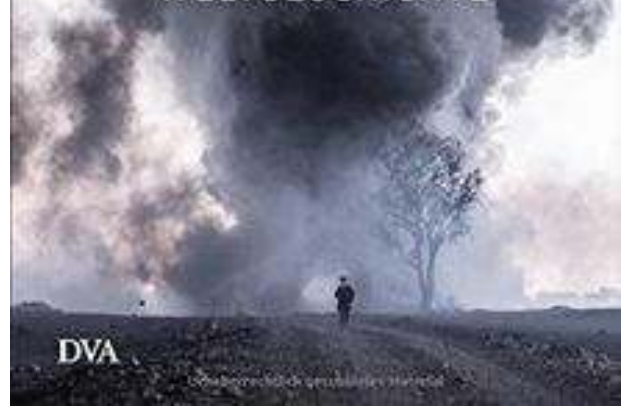
entrainée.

Pour cette raison, l'auteur a structuré son propos à la fois chronologiquement et à partir des catégories de conflits ou de relations conflictuelles observables pendant (ou depuis) une période précise. Du putsch (p. 13-32) à la terreur (p. 241-263), en passant entre autre par l'intervention (p. 149-169) et les meurtres politiques comme ethniques (p. 215-239) comme manières de mener des affrontements, Gregor Schöllgen présente 
au lecteur à la fois un descriptif des conflits - guerres mondiales, de Corée, froide, etc. ainsi qu'une contextualisation et un repositionnement dans le mode de pensée dominant au moment de ces luttes. Comme l'accumulation des ces expériences et l'évolution du cadre cognitif forment les bases sur lesquelles repose notre compréhension du monde d'aujourd'hui, l'ouvrage et particulièrement instructif non seulement pour le public qui s'intéresse à l'histoire du XXème siècle, mais aussi pour celui qui veut connaître certains des fondamentaux de notre présent.

L'ouvrage est facile à lire, avec un langage à la fois simple et précis, et présente au meilleur sens du terme un exercice de vulgarisation scientifique. La typographie et la mise en page correspondent à cela, avec les notes de bas de page - qui peuvent d'ailleurs en soit présenter un intérêt pour les étudiants de l'histoire contemporaine, s'ils veulent avoir un bon point de départ pour la recherche de littérature - placés à la fin du livre. Les deux registres du livre, un pour les personnes et un pour les lieux, sont également très utiles.

Gregor Schöllgen mêle dans son récit l'histoire globale et des exemples de conflits pour lesquels il propose une analyse assez détaillée. Ce jeu d'échelle entre visions " macro " et « micro » est bénéfique à l'ouvrage, et c'est aussi cela qui permet à l'auteur - qui a longtemps instruit les aspirants du service diplomatique allemand - de présenter bien plus qu'une histoire de la guerre. In fine, ce qu'il offre au lecteur est un cours de géopolitique. Le lecteur comprendra par exemple mieux les répercussions des conflits en Asie du sud-est dans les années 1950 pour les deux États allemands (p. 129-131) ou bien le poids de «l'anatomie » de l'URSS dans ses choix d'actions dans les années 1980 (p. 184-186). De même, l'auteur est soucieux de ne pas opérer de catégories simplistes, ni pour les conflits, ni pour les acteurs qu'il décrit.

Tout au long de son exposé des cent dernières années de conflictualité, Gregor Schöllgen opère un deuxième va-et-vient - lui aussi réussi -, celui entre exemples historiques et conséquences pour notre ère. L'auteur l'explique par exemple pour l'échec de l'Europe de la défense en 1954 et ses conséquences pour la capacité de gestion de crise de l'UE en 2008 ou 2015 (p. 133-134) ainsi que pour la genèse des Nations-Unies et l'avènement de régimes de contrôle internationaux des tests nucléaires et les conséquences sur la très difficile question d'une réforme de nos régimes multilatéraux actuels (p. 110-115).

Un autre grand atout de l'ouvrage à signaler ici est que le découpage qu'a choisi Gregor Schöllgen pour son récit ne correspond pas simplement aux dates-clef qu'acceptent la plupart des manuels d'histoire destinés à un grand public. L'auteur structure sa démarche non (seulement) à partir des dates de conflits, mais à partir de changements des concepts du conflit qui ont pu intervenir avec ou suite à la guerre en question. La dimension de communication politique - et le changement de paradigme que cela a occasionné - des bombes atomiques lancées sur Hiroshima et Nagasaki (p. 103-104) est un exemple déjà plutôt connu, celui de la guerre gréco-turque 1920-23 et de l'acceptation quasi-mondiale de déplacements ethniques comme mécanisme de " management de crises et de conflits » (p. 65) qu'elle entraîna l'est peut-être moins.

Le bilan que l'auteur dresse à la fin de son ouvrage (p. 327-338) est d'un grand intérêt : il résume sur une dizaine de pages ce qui façonne notre monde contemporain, et cela mieux que bien des écrits émanants de la plume d'analystes «de l'actualité géopolitique ». Que ce soit par intérêt pour l'évolution du monde à travers un XXème siècle particulièrement violent ou que ce soit par intérêt pour les fondamentaux 
politiques du XXIème siècle que nous vivons, cet ouvrage offre au lecteur des renseignements d'une grande valeur.

INDEX

Thèmes : Histoire militaire

Index chronologique : Contemporaine

AUTEUR

PHILIPP SIEGERT

IFRA-SHS 